\title{
Original
}

CODEN-JHTBFF, ISSN 1341-7649

\section{Expression and Clinical Significance of Matrix Metalloproteinase-2 and Its Inhibitor TIMP-2 in Oral Squamous Cell Carcinoma}

\author{
Qu Hong ${ }^{1)}$, Tan Jun ${ }^{2)}$, Jin Lei ${ }^{3)}$, Jing Xiling ${ }^{4)}$ and Ryo Tamamura ${ }^{5)}$ \\ ${ }^{1)}$ DaLian Medical University Stomotological College, China \\ ${ }^{2)}$ The Second Affliated Hospital Of DaLian Medical University, China \\ ${ }^{3}$ DaLian University Stomatological College, China \\ ${ }^{4)}$ DaLian Medical University Stomotological College,China \\ ${ }^{5)}$ Department of Oral Pathology and Medicine, Graduate School of Medicine, Dentistry and Pharmaceutical Sciences, Okayama \\ University, Japan. \\ (Accepted for publication, march 16, 2006 )
}

\begin{abstract}
Oral squamous cell carcinoma is the most common malignant tumour in the head and neck area, and is considered as the top oral carcinoma. A large number of studies have determined that many matrix matalloprpteinases (MMPS), especially MMP-2 and MMP-9 are eminent, correlative with Oral squamous cell carcinoma (OSCC)'s invasion and metastasis. However, the research on the expression of MMP-2 and its inhibitor (TIMP-2) in oral squamous cell carcinoma is very rare. Here we examine the expression pattern of MMP-2 and TIMP-2 in 10 normal oral mucosa, 10 dysplastic oral mucosa and 30 oral squamous cell cancer tissues, using immunohistochemical method in order to investigate the correlativity and the effect to the biological behaviour of Oral Squamous Cell Cancer. We found that the difference of MMP-2 expression in oral squamous cell cancer tissues, and dysplastic oral tissues versus normal oral tissues was statistically significant. There is a close correlation between the MMP-2 expression level and the carcinoma differentiation, depth of invasion, lymph node metastasis, and distant metastasis. The expression of TIMP-2 in oral squamous cancer was higher than in normal oral mucosa but lower than in dysplastic mucosa. The difference of the expression of TIMP-2 in positive and negative lymphnode metastasis was significant. There is an inverse correlation between the MMP2 expression and the TIMP-2 expression in oral squamous cell carcinoma tissues $(r=3.89 \mathrm{p}<0.01)$. In the process of invasion and metastasis of OSCC, imbalance between MMP-2 and TIMP-2 expression plays an important role. Detecting MMP-2 and TIMP-2 protein expression may be considered as markers for estimating the biological behavior of carcinoma and evaluating the prognosis of patients.
\end{abstract}

Keywords: Immunohistochemistry. Matrix Metalloproteinase-2 (MMP-2) Oral squamous cell carcinoma, Tissue inhibitors of metalloproteinase-2 (TIMP-2)

\section{Introduction}

The invasion and metastasis of a tumor cell can be separated into three steps: attachment, matrix dissolution, migration. Only when the three processes join in a line can direct invasion come true. This process consists of a series of tumor-host interactions that involve multiple extracellular matrix degrading enzymes, including serine proteinases, cysteine proteinases, and matrixmetalloproteinases (MMPs) ${ }^{1-3}$. . MMPs play an important role in the malignant behavior of the neoplasm ${ }^{3}$. They are a family of Zinc-dependent proteinases that can degrade all components of extracellular matrix (ECM). This family of endopeptidases is associated with ECM degradation in physiological and pathological conditions such as embryonic development, wound healing, angiogenesis, arthritis, inflammation, and tumor

Corresponding author: Qu Hong, DaLian Medical University Stomotological College No .465 ZhongShan Road DaLian, 116027, ChinaTel.:+86-0411-84720075, Fax. : +86-0411-84751086 metastasis. They are classified into four groups according to their substrate specificity: collagenases (MMP-1, -8, and -13), gelatinases (MMP-2 and -9), stromelysins (MMP-3, -10, and membrane-type MMPs (MT-MMPs) (MMPs 14-17, 24, and 25). Alignment of the amino acid sequences between these enzymes shows that there is a high degree of homology between the enzymes in each group(about 80\%) and between groups (about $50 \%)^{3-6)}$.

Many studies has demonstrated that the increased expression of MMP-2 and MMP-9 is closely related to the invasion and metastasis of oral squamous cell carcinoma. However, the conclusions are incongruous. Though MMP-2 expression in oral squamous cell carcinoma has been reported, few reports have been shown about both MMP-2 and TIMP-2 in oral squamous cell carcinoma and the relation in the invasion and metastasis of oral squamous cell cancinoma has not been reported yet. The aim of 
Qu Hong et al.: Matrix Metalloproteinase-2 and Its Inhibitor TIMP-2 in Oral Squamous Cell Carcinoma

the present study is to test the expression of MMP-2, TIMP-2 in human patients with oral squamous cell cancinoma tissues, using the immunohistochemical method, in order to investigate the correlativity and the effect to the biological behaviour of oral squamous cell cancinoma.

\section{Materials and Methods}

\section{Clinical Data}

\subsection{Source of Tissue}

30 random samples were selected from paraffin blocks of primary oral squamous cell cancer tissue which were removed from patients of DaLian Oral Hospital from January 2002 to October 2004. These samples include 17 male cases and 13 female cases. The median age was 59.31. None of the cases had received radiotherapy or chemotherapy. 10 random samples were selected from dysplastic oral mucosa and normal oral mucosa respectively as a control reaction. Review the clinic pathological features of all the oral squamous cell cancer cases.

\subsection{Classification criterion of pathology}

The histology and differentiation of the tumors were determined according to WHO New Histological Classification Of Tumors. The grades of malignant tumors were determined according to differentiation, heterotype and mitosis. More people prefer three grades classification. Those are: GradeI (advanced differentiation); cytological and histological characteristics are similar with normal oral squamous epithelium. The ratios of basal cell and squamous cell which has on intercellular bridge is different. Keratinization is distinct, mitosis is less, dysplasic mitosis and multinucleate tumor cells are rare, the polymorphism of the cell and nucleus is slight. GradeII (middle differentiation). The features of the tumor are in the middle of gradeI and grade III. Comparing with gradeI, keratinization is less, the polymorphism of cell and nucleus increases, the rate of mitosis increases, occasionally, abnormal mitosis is present, intercellular bridge is unclear. Grade III (poor differentiation): cytological and histological characteristics are slightly similar with normal oral squamous epithelium. Keratinization is rare. There is very little intercellular bridge, abnormal mitosis is frequently observed. With the The polymorphism of cell and nucleus, the number of multinucleate tumor cell increases. The malignancy of grade I and grade II is low. If the tumor shows the features of different grades, the classification is determined by the high malignant one. Dysplastic oral mucosa refers to the epithelium which has some degree of heterotype, but this is not enough to diagnose cancer. The histological features of dysplastic epithelium can be described as follows: Basal cell polarity is lost, more than one stratification of basal cell present, an increased nuclear/cytoplasmic ratio, abnormal stratification of epithelium, increase in the rate of mitosis and abnormal mitosis is present. There is mitosis in the upper $1 / 2$ stratification of epithelium. Polymorphism of cell and nucleus, nucleus is darkly strained, cell nucleus becomes bigger, the adhension of cell to cell is lost, in the stratification of the pricle cell, a single or a group of cutinized cell is present.

\section{Methods}

\subsection{Main Reagents}

Mouse anti MMP-2 and TIMP-2 monoclonal antibody, SP immunohistochemical reagents provided by Shanghai Biological Science and Technique Ltd.

\subsection{Staining Method of Immunohistochemistry}

Tissues labeling reactions for MMP-2 and TIMP-2 were sequentially done as follows:

Paraffin-embedded tissues were dewaxed in xylen and rehydrated through graded alcohol.

The sections were treated with methanol-hydrogen peroxide complex solution for 30 minutes so that endogenous dioxygenase was lost. Washed with PBS Solution $(2 \mathrm{~min} \times 3)$. The section was incubated with first antibody (the first antibody was diluted with PPS to 1:100) for $1 \mathrm{hr}$ at room temperature in a moist chamber. After washing with PBS, incubated with the second antibody(the antibody was diluted with PPS to $1: 100$ ) for $30 \mathrm{~min}$ at room temperature discontinuously. Added PAB Solution and observed the color reaction under a microscope, stopped the color reaction timely with water, the reaction continued not more than $10 \mathrm{~min}$. Counter stained nucleus with haematoxylin or methyl green for $10 \mathrm{sec}$. Dehydrated with gradient alcohol, made it transparent with xylene, fixed with neutral gum.

\subsection{Result Determination}

There were light brown granules in the cytoplasm of MMP-2 and TIMP-2 positive cells. If both tumor cells and fibroblasts mononucleophases in matrix showed positive granules, the reaction was determined as positive one. If fibroblasts and mononucleophases were positive but the tumor cell was negative. The reaction was determined as negative one. In each sections, five fields of high magnification were selected. According to the percentage of positive cell to all the squamous epithelium cell, they were classified as follows: less than 30\% (+); 30-60\% (++); more than $60 \%(+++)$. KL computer assisted immune image analysis system was used to determine the result.

\section{Statistical Analysis}

All the data was denoted as $\mathrm{X} \pm \mathrm{S}$, SPSS10.0 software was used to carry out date statistics. $\mathrm{X}^{2}$-test or Fisher's exact probability was used to determine the significant difference.

\section{Results}

1. The expression of MMP-2, TIMP-2 in normal oral mucosa, dysplastic oral mucosa and oral squamous cell cancinoma tissue. 


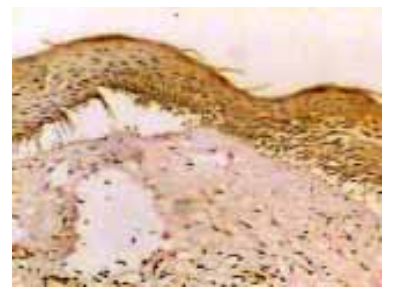

Fig.1. the expression of MMPin oral mucosa epithelium (-) (x100) 2

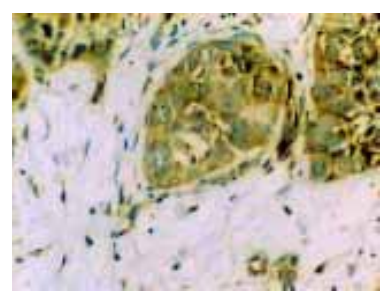

Fig.3 the expression of MMP-2in advanced differentiation oralcell cancer $(+)(x 200)$

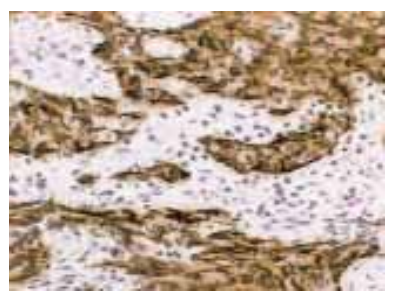

Fig .5 the expression of MMP-2 in poor differentiation oral squamous cell cancer $(+++)$ (x200)

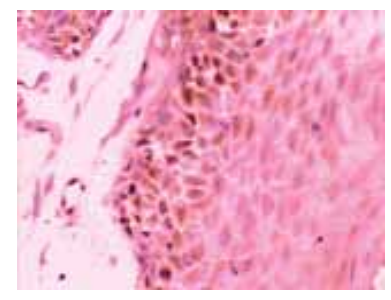

Fig.2. the expression of MMP-2 in dysplastic oral mucosa $(+)$ (x200)

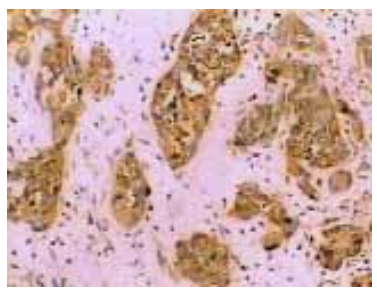

Fig.4 the expression of MMP-2 in middle differentiation oral squamous cell cancer $(++)$ (x100)

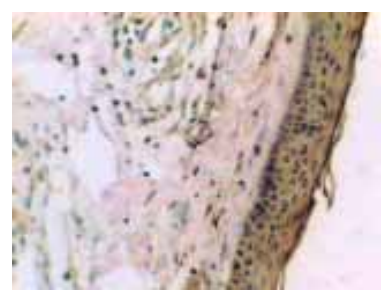

Fig. 6 the expression of TIMP-2 in oral mucosa epithelium (-) (x100)

\subsection{Expression of MMP-2}

MMP-2 was mainly expressed in the cytoplasm of tumor cell. It could be observed as light brown granules under high magnification microscope. The expression of normal oral mucosa epithelium was negative and low staining indicating negative (Fig .1). The expression of dysplastic oral mucosa epithelium was higher (Fig .2). The expression of oral squamous cell cancer varied from low to high positive (Fig .3.4.5). From normal oral mucosa epithelium, dysplastic oral mucosa epithelium to oral squamous cell cancer, the expression of MMP-2 increased. The difference of oral squamous cell cancer and normal oral mucosa epithelium, dysplastic oral mucosa epithelium and normal oral mucosa

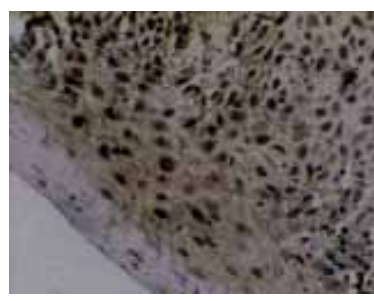

Fig .7 the expression of TIMP-2in dysplastic oral mucosa $(++)$ (x200)

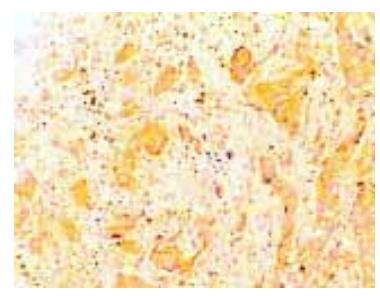

Fig.9 the expression of TIMP-2in middle differentiation oralsquamous cell cancer $(+)$ (x100)

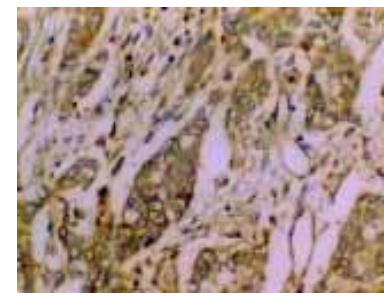

Fig .8 the expression of TIMP-2 in advanced differentiation oral squamous cell cancer $(+)(\mathrm{x} 200)$

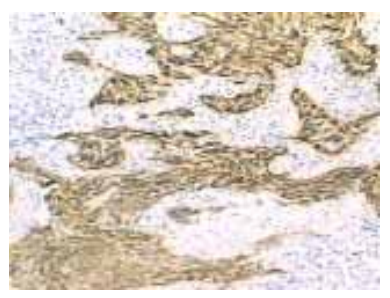

Fig.10 the expression of TIMP-2 in poor differentiation oral squamous cell cancer $(++)(x 100)$ epithelium was significant $(\mathrm{p}<0.01)$. The difference of oral squamous cell cancer and dysplastic oral mucosa epithelium was not significant $(\mathrm{p}>0.05)$.

\subsection{The expression of TIMP}

The positive expression of TIMP was also detected in the cytoplasm as light brown granules. The expression of TIMP in normal oral mucosa epithelium was negative or low positive (Fig $.6)$, the expression in dysplastic oral mucosa epithelium varied from low positive to high positive (Fig .7), the expression in oral squamous cancer varied from low positive to high positive (Fig .8.9.10).

\section{The relation between MMP-2, TIMP expression and patients' clinicopathological features.}

\subsection{The relation of the expression and patients' age and sex .}

From table-II, the positive percentages of MMP-2 in male were $34.7 \pm 5.2$ and in female were $35.2 \pm 5$.6. The positive percentages of TIMP-2 in male were $9.9 \pm 4.6$, in female were $9.8 \pm 5.1$. In patients whose age was above or equal to 50 and below 50 , the positive percentage of MMP-2 was $34.6 \pm 5.6$ and $33.5 \pm 4.2$ respectively, the positive percentage of TIMP-2 was $9.9 \pm 5.2$

Table 1 The expression of MMP-2, TIMP-2 in normal oral mucosa, dysplastic oral mucosa and oral squmous cell cancer

\begin{tabular}{lccc}
\hline Variables & Number of patients & MMP-2 (\%) & TIMP-2 (\%) \\
\hline Oral squamous cell cancer & 30 & $35.5 \pm 5.6$ & $9.8 \pm 5.5$ \\
dysplastic oral mucosa & 10 & $32.4 \pm 5.5(1)$ & $10.2 \pm 5.5(3)$ \\
normal oral mucosa & 10 & $8.2 \pm 5.5(2)$ & $6.9 \pm 5.6(4)$ \\
\hline
\end{tabular}

(1) Level of MMP-2 oral squamous cell cancer vs dysplastic oral mucosa p>0.05 (2) Level of MMP-2 oral squamous cell cancer vs normal oral mucosa $\mathrm{p}<0.01$ (3) Levels of TIMP-2, oral squamous cell cancer vs dysplastic oral mucosa $\mathrm{p}>0.05$ (4) Levels of TIMP- 2, oral squamous cell cancer vs normal oral mucosa $\mathrm{p}<0.01$ 
Qu Hong et al.: Matrix Metalloproteinase-2 and Its Inhibitor TIMP-2 in Oral Squamous Cell Carcinoma

and $9.7 \pm 4.5$ respectively. The difference of the expression of MMP-2 and TIMP-2 in oral squamous cancer in different age and sex was not significant $(\mathrm{P}>0.05)$.

\subsection{The relation between the expression and tissue's position .}

From table-II, according to occurrence position, the positive percentages of MMP-2 were listed as follows: tongue: $35.3 \pm 4.2$, gingiva: $35.1 \pm 4.5$, cheek: $33.7 \pm 4.5$. The positive percentages of TIMP-2 were as follows tongue: $9.8 \pm 5.1$, gingiva: $9.8 \pm 5.2$, cheek: $9.9 \pm 4.6$. The difference of the expression in oral squamous cancer in different position was not significant $(\mathrm{P}>0.05)$.

\subsection{The relation of the expression and tumor's differentiation.}

The positive percentages of the expression of MMP-2 in advanced, middle, and poor differentiation oral squamous cell cancer were $34.8 \pm 5.2,35.2 \pm 5.7$, and $35.3 \pm 5.2$. The difference was not significant $(\mathrm{P}>0.05)$. The positive percentages of the expression of TIMP in advanced, middle, and poor differentiation oral squamous cancer were; $9.8 \pm 4.6,9.7 \pm 5$.2, and $9.8 \pm 5.2$.The difference was not significant.

2.4 The relation between the expression of MMP-2,TIMP-2 and tumor invasion depth

In the superficial and deep muscular layer the positive percentages of the expression of MMP-2 were $28.7 \pm 4.6,37.4 \pm$ 4.2 respectively. The positive percentages of the expression of TIMP-2 were $12.5 \pm 1.5,7.1 \pm 2.6$ respectively. The difference was significant $(\mathrm{P}<0.01)$.

2.5 The relation between expression MMP-2, TIMP-2 and

\section{lymphnode metastasis.}

In this study, the positive percentages of the expression of MMP2 in positive, the lymphnode metastasis was $39.2 \pm 5.2$, but in negative are lymphnode metastasis, the positive percentage was $30.1 \pm 4.5$. The difference was significant $(\mathrm{P}<0.01)$. The positive percentage of the expression of TIMP-2, in positive lymphnode metastasis was $8.2 \pm 1.6$, but in negative lymphnode metastasis, the positive percentage was $13.1 \pm 2.2$, The difference was significant $(\mathrm{P}<0.01)$.

3. The correlation between the expression of MMP-2 and the expression of TIMP-2 in oral squamous cell cancer (table-III).

Twenty of the thirty oral squamous cell cancer cases displayed positive expression of MMP-2, eighteen of the thirty cases displayed positive expression of TIMP-2, fourteen cases displayed positive expression of both MMP-2 and TIMP-2, four cases displayed negative expression of both MMP-2 and TIMP-2. According to the statistical analysis, there was an inverse correlation between the MMP-2 expression and TIMP-2 expression in oral squamous cell cancer tissues $(\mathrm{r}=3.89, \mathrm{P}<0.01)$. We draw the conclusion that : 1 . In the development of normal oral mucosa dysplastic oral mucosa; oral squamous cell cancer tissues, the expression of MMP-2 in creased. Namely, the expression of MMP-2 in oral squamous cell cancer was higher than in dysplastic oral mucosa, the expression of MMP-2 in dysplasic oral mucosa is higher than in normal oral mucosa. This indicate that the stroma cell had the capacity of secreting MMP-2 in the precancer stage. The difference of the expression of MMP2 in different grades was not significant, but between the positive and negative lymphnode metastasis, the different was significant.

Table 2 The relation between the expression of MMP-2, TIMP-2 and patient's clinicopathological features .

\begin{tabular}{|c|c|c|c|c|c|}
\hline variables & cases & MMP-2 (\%) & $\mathrm{P}$ & TIMP-2 (\%) & $\mathrm{P}$ \\
\hline \multicolumn{6}{|l|}{ sex } \\
\hline male & 17 & $34.7 \pm 5.4$ & & $9.9 \pm 4.6$ & \\
\hline female & 13 & $35.2 \pm 5.6$ & $>0.05$ & $9.8 \pm 5.1$ & $>0.05$ \\
\hline \multicolumn{6}{|l|}{ age } \\
\hline $\mathrm{R} 50$ & 15 & $34.6 \pm 5.6$ & & $9.9 \pm 5.2$ & \\
\hline$<50$ & 15 & $33.5 \pm 4.2$ & $>0.05$ & $9.7 \pm 4.5$ & $>0.05$ \\
\hline \multicolumn{6}{|l|}{ position } \\
\hline tongue & 14 & $35.3 \pm 4.2$ & & $9.8 \pm 5.1$ & \\
\hline gingiva & 9 & $35.1 \pm 4.5$ & $>0.05$ & $9.8 \pm 5.2$ & $>0.05$ \\
\hline cheek & 7 & $33.7 \pm 5.4$ & & $9.9 \pm 4.6$ & \\
\hline \multicolumn{6}{|l|}{ Differentiation degree } \\
\hline advanced & 16 & $34.8 \pm 5.2$ & & $9.8 \pm 4.6$ & \\
\hline middle & 10 & $35.2 \pm 5.7$ & $>0.05$ & $9.7 \pm 5.2$ & $>0.05$ \\
\hline poor & 4 & $35.3 \pm 5.2$ & & $9.8 \pm 5.2$ & \\
\hline \multicolumn{6}{|l|}{ Invasion depth- } \\
\hline Mucous and superficial muscular & 24 & $28.7 \pm 4.6$ & & $12.5 \pm 1.5$ & \\
\hline Deep muscular and under muscular tissue & 6 & $37.4 \pm 4.2$ & $<0.01$ & $7.1 \pm 2.6$ & $<0.01$ \\
\hline \multicolumn{6}{|l|}{ Lymphnode matastasis } \\
\hline positive & 11 & $39.2 \pm 5.2$ & & $8.2 \pm 1.6$ & \\
\hline negative & 19 & $30.1 \pm 4.5$ & $<0.01$ & $13.1 \pm 2.2$ & $<0.01$ \\
\hline
\end{tabular}


Table 3 The correlation between the expression of MMP-2 and the expression of TIMP -2 in oral squamous cell cancer

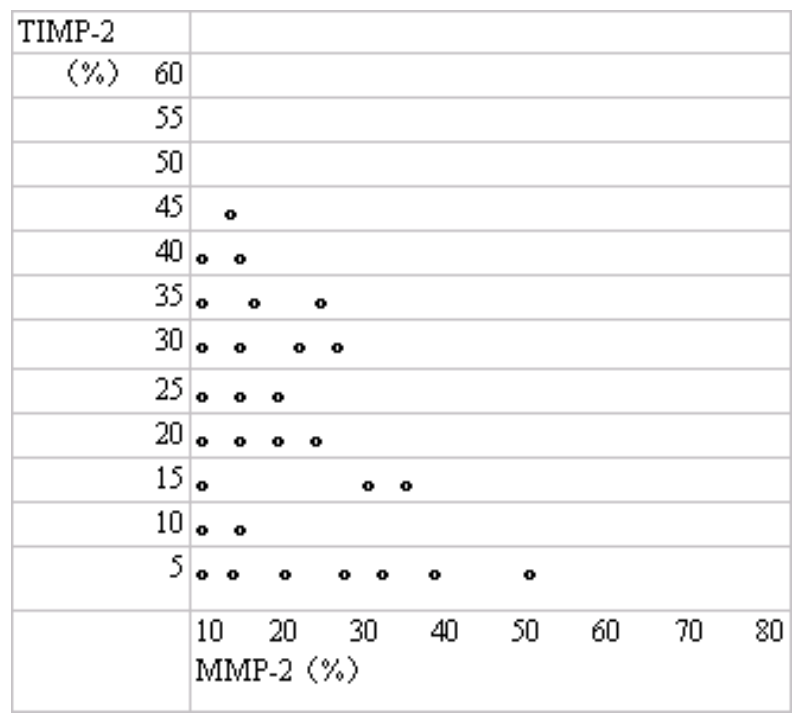

High levels of MMP-2 may be the moleculor base for increasing invasion and metastasis capacity of oral squamous cell cancer. 2. The expression of TIMP-2 in oral squamous cancer was higher than in normal oral mucosa but lower than in dysplastic mucosa. The difference of the expression of TIMP-2 in positive and negative lymphnode metastasis was significant.

3. There was an inverse correlation between the MMP-2 expression and the TIMP -2 expression in oral squamous cell cancer tissues From the advanced differentiation squamous cancer to middle differentiation, squamous cancer to poor differentiation squamous cancer, the ratio of MMP-2 to TIMP-2 showed a rising trend, indicating that the ratio of MMP-2 to TIMP-2 was a more valuable index for a tumor's invasion, metastasis and prognosls.

4. The levels of the expression of MMP-2, TIMP-2 was a good predictor of the biological behavior of oral squamous cell cancer. It was a new target for the therapy of stubbon and recurrent oral squamous caner.

\section{Discussion}

In this study we have found that MMP-2 is mainly expressed in the cytoplasm of tumor cells, but also expressed in fibroblasts and mononucleophages. In the area between normal tissue and in the invasion edge of the tumor, there is an obviously high expression of MMP-2, indicating that the MMP-2 is closely related to tumors invasion and metastasis. Westermarck ${ }^{6}$ suggested that the tumor cells may interact with stromal cells via soluble medium or membrane -associated molecule, producing and regulating MMP-2, facilitating tumor's invasion and metastasis. The positive expression of MMP-2 is increased in oral squamous cell cancer and dysplastic oral mucosa.

The explanation is probably under normal conditions, tissue synthesize and secret little MMP-2. However, under abnormal conditions such as inflammation, hyperplasia injury, tissue synthsize and secret more MMP-2. Therefore, increasing expression of MMP-2 can be shown in the precancerous lesions or in the earlier stage of oral squamous cancer. Activation of MMP2 gene may be the main factors related to oral squamous cell cancer's occurrence and progress. Inhibiting the expression of MMP-2 and it's biological activity may be a valuable method to prevent and manage oral squamous cell cancer.

In recent years, the relationship between the expression of MMP2 and tumor's PT stage, histology grade, and prognosis has been examined, using a variety of methods, but the conclusions are incongruous. Some investigators ${ }^{7-9)}$ suggested that the increasing of MMP-2‘s expression and activity was closely related to the lymphnode metastasis of oral squamous cell cancer, but it was not related to the tumor's PT stage and histology grade. Giannell ${ }^{10)}$ found that: As a structural protein of ECM. LN-5 can be cleaved by MMP-2 at the $\mathrm{V}_{2}$ chain which is between a long arm and a short arm, then it will become a soluble fragment. Based on this, MMP-2 induces the migration of in flammatory cells and tumor cells, and plays an important role in the regulation of a tumor's migration. This study demonstrated that the differences of the expression of MMP-2 in different degree differentiation of oral squamous cell cancer is not significant. But the expression in positive lymphnode metastasis is clearly higher than in negative lymphnode, indicating the probability that with a higher expression of MMP-2, tumor cells will invade blood and lymphatic vessel, than the mefastascs is realined. This is agreement with the earlier studies ${ }^{11-15)}$.

Forsy et al. ${ }^{11)}$ addressed MMP-2 has a capacity of activiting MMP-9 which can induce tumor angiogenesis in the invasion and metastasis of a later stage tumor.

Juan Carlos de Vicente ${ }^{16)}$ examined MMP-2 and MMP-9 in OSCC, using immunohistochemistry, suggested that the expression of MMP-2 and -9 measured by immunohistochemistry is related to the invasion of OSCC, and MMP-9 is related to a poor prognosis in a subset of patients without neck node metastasis. Keizo Kato ${ }^{17)}$ suggested that combination analysis of Zymography and FIZ is considered to be a useful method for detecting the activity as well as the localization of MMPS in human OSCC.

Richard C.K.Jordan ${ }^{18}$ had used quantitative reverse transcription -PCR to show that elevated levels of MMP-1 and -9 mRNA are significantly associated with oral dysplasias that progressed to oral cancer compared with those dysplasias that did not progress; levels of MMP-1 and -9 mRNA may be markers of malignant transformation at this site.

In the process of a tumor's invasion and metastasis, the secretion and activation of metalloproteinases are not enough to ensure that they will degrade the target matrix substrate. This is because that the natural tissue inhibitors of metalloproteinases (TIMP) can block matrix metalloproteinases (MMP), activity by two distinct mechanisms.

1) In the zymogen activation stage, TIMP-2 binds tightly to the 
Qu Hong et al.: Matrix Metalloproteinase-2 and Its Inhibitor TIMP-2 in Oral Squamous Cell Carcinoma

zymogen of MMP-2 (pro MMP-2) forming a complex, while TIMP-1 forms a specific complex with proMMP-9. Thus TIMP inhibite proMMP activation.

2) TIMPs bind to activated MMPS forming a 1:1 complex. For example, TIMP-1 and TIMP-2 can directly bind to activated MMP forming a tight 1:1 complex. It is worth noticing that recombinant TIMP can also suppress MMP activity. In this study, we found comparing with normal oral mucosa, the expression of TIMP doesn't decrease but increase. The probable reasons are as follows: (A) In the complex of TIMP-2 and MMP-2, the ratio of TIMP -2 to MMP-2 is $1: 1$, so the host will suppress the MMP-2 by regulating the expression of TIMP-2.

(B) TIMP-2 is the inhibitor of MMP-2. TIMP-2 can bind to not only the activated MMP-2 but also the zymogens of MMP-2 and inhibits hydrolysis activity of all the members of MMP family ${ }^{13)}$ When cancer occurs, with increasing expression of other MMPS, the expression of TIMP also increases.

(C) There may be other unknown factors which are valuable to study further.

Recent studies demonstrated that TIMP-2 inhibits bFGFinduced human microvascular endothelial cell proliferation but TIMP-2 and a newly synthetic metalloproteinase inhibitor (BB94) does not have this function. Judging from above, TIMP effect this function not by inhibiting MMP activity but by directly blocking the growth. This is an additional activity of TIMP-2.This finding shows that TIMP-2 can not only prevent the tumor's invasion and metastasis by suppressing the degradation but also block the continued growth of primary tumor by inhibiting bFGFinduced human micro-vascular endothelial cell proliferation. A single study showed that TIMP-2 was associated with regional lymph node and distant metastasis and poor prognosis. In particular, a marked expression of TIMP-2 was the most important indicator and is valuable in identifying the patients at high risk for poor prognosis even in early clinical stage ${ }^{19)}$. Our present study has found the expression of TIMP is not related to a patient's age, sex and tumor's degree of differentiation, but closely related to a tumors lymph node metastasis. However, if the expression of TIMP-2 can be used as an independent predictor of the biological behavior of oral squamous cell cancer needs to be investigated further.

The balance between the MMP and the TIMP is crucial for the synthesization and degradation of ECM. There is a delicate balance between the MMP and the TIMP in normal tissue to maintain the stability of ECM. When cancer occurs, the expression of the MMP, and the TIMP increases.If the expression of the TIMP is not high enough to inhibit the MMP, the balance will be destroyed, the MMP activity will increase. BM and ECM will be degraded. Then the invasion and metastasis of tumor will be realized. In this study we found the expression of MMP -2 was higher than that of TIMP-2, and there was an inverse correlation between the MMP-2 expression and the TIMP-2 expression in oral squamous cell cancer $(\mathrm{p}<0.01)$.

During the invasion and metastasis of tumors, disruption of this balance may lead to the degradation of ECM. Therefore, as far as the malignant tumor is concerned, MMP/TIMP may be a more valuable prognostic indicators of the invasion and metastasis of tumor. Gohji et $\mathrm{al}^{15)}$ found that the MMP/TIMP increased in invasive bladder cancer. Its malignancy is high, recurrence is early and prognosis is poor. Investigating and developing externaly active and recombinant TIMP-2 will provide a novel and efficient way to manage oral squamous cell. The transfection of TIMP-2 gene may enhance the expression of TIMP-2 and its capacity of inhibiting the secretion and activation of MMP-2, providing a reference frame for gene therapy of oral squamous cell cancer, preventing the invasion and metastasis of it, improving prognosis.

To sum up, the expression of MMP/ TIMP has a great potential value in the assessment of the progress and metastasis potency and prognosis of oral squamous cell cancer. Multiple genes are involved in the expression of MMP/TIMP, The precise control mechanisms have not been elucidated, the studies are still in an initial stage. We believe that with the development of biology and the continue investigations in this field. The research of MMP and TIMP will play a more important role in both basic science and clinical therapy, providing a more directional target for anticancer therapy.

\section{Acknowledments}

This work was partially supported by grants in aid for scientific researches from the Ministry of Edition, Culture, Sports, Science and Technology (\#15209060,17406027, 17591910, 17591911).

\section{References}

1. Liu L et al. The function of MMP-2.9 in oral squamous cell cancer's invasion and metastasis. Foreign Medicine (Stomatology) 31(1): 20, 2004.

2. WHO New Histological Classification of Tumors 77-81, 2001.

3. Liotta LA,Tryggvason K, Garbisa S, et al. Metastatic potential correlates with enzymatic degradation of basement membrane collagen. Nature 284:67-68, 1980.

4. Davies B, Miles DW, Happerfield LC, et al. Activity of type IV collagenases in benign and malignant breast disease. Br J Cancer 67:1126-1131, 1993.

5. Davies B, Waxman J, Wasan H, et al. Levels of matrix metalloproteinase in bladder cancer correlate with tumor grade and invasion. Cancer Res 53:5365-5369, 1993.

6. Westermarck J, Kahari VM. Regulation of matrix metalloproteinase expression in tumor invasion. FASEB J 13(8):781, 1999.

7. Maekawa K, Sato H, Furukawa M, et al. Clin Exp Metastasis 19(6):513-518, 2002.

8. Yorioka CW, Coletta RD, Alves F, et al. Int J Oncol 20(1):189194, 2002. 
9. Coletta RD, Cotrim P, Vargas PA, et al. Oral Surg Oral Med Oral Pathol Oral Radiol Endod 91(5):563-569, 2001.

10. Giannelli G, Falk-Marzillier J, Schiraldi O, et al. Induction of cell migration by matrix metalloprotease- 2 cleavage of laminin-5 [J]. Science 277(5323):225, 1997.

11. Forsyth PA, Wong H, Laing TD, et al. Br J Cancer 79(1112):1828-1835, 1999.

12. Brew K, Dinakarpandian D, Nagases H.Tissue inhibitors of metalloproteinases: evolution,structure and function. Biochem Biophys Acta 1477(1-2):267, 2000.

13. Liotta LA, Stetler-Stevenson WG. Trmor invasion and metastasis: an imbalance of positive and negative regulation. Cancer Res 51(18 Suppl):5054, 1991.

14. Murphy AN, Unsworth EJ, Stetler-Stevenson WG. Tissue inhibitor of metalloproteinase-2 inhibits bFGF-induced human microvascular endothelial cell proliferation. J Cell Physiol 157(2):351, 1993.

15. Gohji K, Fujimoto N, Ohkawa J, et al. Imbalance between serum matrix metalloproteinase-2 and its inhibitor as a predictor of recurrence of urothelial cancer. Br J Cancer 77(4):650, 1998.
16. Juan Carlos de Vicente, Manuel Florentino Fresno, Lucas Villalain, Jose' Antonio vega, Gonzalo Hernandez Vallejo, et al. Expression and clinical significance of matrix metalloproteinase- 2 and matrix metalloproteinase- 9 in oral squamous cell carcinoma. Oral Oncology 41, 283-293, 2005.

17. Keizo Kato, Akira Hara, Toshiya Kuno, Nami Kitaori, Zhi Huilan, Hideki Mori, Makoto Toida and Toshiyuki Shibata, et al. Matrix metalloproteinases 2 and 9 in oral squamous cell carcinomas: manifestation and localization of their activity. Journal of Cancer Research and Clinical Oncology.

18. Richard C. K. Jordan, Maricris Macabeo-Ong, Caroline H. Shiboski, Nusi Dekker, David G. Ginzinger, David T. W. Wong, and Brian L. Schmidt. Overexpression of Matrix Metalloproteinase-1 and -9 mRNA IsAssociated with Progression of Oral Dysplasia to Cancer. Clinical Cancer Research 10: 6460-6465, 2004.

19. Akihiro Katayama, Nobuyuki Bandoh, Kan Kishibe, Miki Takahara, Takeshi Ogino, Satoshi Nonaka, and Yasuaki Harabuchi. Expressions of Matrix Metalloteinases in EarlyStage OralSquamous Cell Carcinoma as Predictive Indicators for Tumor Metastases and Prognosis. Clinical Cancer Research 10: 634-640, 2004. 